\title{
Physico-chemical Analysis of Flexible Polyurethane Foams Containing Commercial Calcium Carbonate
}

Sabrina Sá e Sant’Anna a Denilson Arlindo de Souza a, Danielle Marques de Araujo,

\author{
Cornélio de Freitas Carvalho ${ }^{\mathrm{b}}$, Maria Irene Yoshida ${ }^{\mathrm{a}}$
}

${ }^{a}$ Department of Chemistry, UFMG,

Av. Antônio Carlos, 6627, Pampulha, 31270-901 Belo Horizonte - MG, Brazil

${ }^{\mathrm{b}}$ Department of Chemistry, Campus Universitário, UFOP,

Morro do Cruzeiro, 35400-000 Ouro Preto - MG, Brazil

Received: May 28, 2008; Revised: December 19, 2008

\begin{abstract}
Calcium carbonate $\left(\mathrm{CaCO}_{3}\right)$ is a filler often utilized by the Brazilian mattress factories in the production of polyurethane foams. The filler allows the substitution of part of the polymeric agents, conferring dimensional stability and hardness to the foams. However, in agreement with experimental data, it is observed that the excess of commercial $\mathrm{CaCO}_{3}$ utilized in industry causes the increase of hysteresis, possibly causing permanent deformations and damaging the quality of the final product. In the present work, the physico-chemical analyses of the flexible polyurethane foams with different contents of $\mathrm{CaCO}_{3}$ were performed. The foams are submitted to the morphological, mechanical and positron analyses to verify the alterations provoked by the progressive introduction of this filler.
\end{abstract}

Keywords: polyurethane, foam, calcium carbonate

\section{Introduction}

The versatility of polyurethane chemistry permits the production of a great variety of materials such depending on the initial ingredients used in the synthesis ${ }^{1}$. Flexible polyurethane foams are one of the most important classes of cellular plastic and can be applied in the fabrication of a wide range of materials for different uses such as foam mattresses, pillows, furniture, cushioning materials for automobiles, packing, recreation, shoes, etc. ${ }^{2}$.

In general, industries that produce flexible polyurethane foams use fillers to modify the material's properties in some way, such as: dimensional stability, retraction from the mold and density ${ }^{3-4}$. When adding a filler to a polymer to form a conjugated biphasic material, the tension applied to the polymeric matrix will be transferred in part to the disperse phase, the filler, since it presents properties superior to the pure polymer ${ }^{5}$. Efficient reinforcement is achieved by interactions of the constituents of the biphasic material ${ }^{6-7}$ via mechanisms of adhesion, which could be: adsorption, electrostatic attraction, chemical bonding and mechanical adhesion.

Chemical bonding is the most efficient form of adhesion and occurs with the application of coupling agents on the surface of the filler, which serves as a bridge between the polymer and the reinforcement. In mechanical adhesion, the polymer fills in the grooves of the filler; this adhesion tends to be low unless there is a large number of recesses on the surface of the filler ${ }^{8}$.

In principle any material can be used as a filler. However, some aspects must be considered when selecting the material for this purpose. These include: size $^{9}$, in that the particles must be small and able to easily disperse in the polymer matrix; chemical purity, to avoid undesired reactions; and abrasiveness, which can cause excessive deterioration to the mixing equipment and increase costs ${ }^{8}$.
Among the inorganic materials utilized as filler, notable ones include: calcium carbonate, aluminum hydroxide, silica, titanium dioxide and $\operatorname{talc}^{6}$. Some of the organic materials more commonly used are carbon black ${ }^{10}$ and natural fibers ${ }^{11-12}$.

In flexible polyurethane foams, the fillers promote an increase in density and resistance to compression. However, they reduce the resiliency and contribute to the increase in permanent deformation. In addition, properties such as tear strength, for example, are significantly affected by the introduction of filler ${ }^{13}$. Accordingly, it is necessary to know the end-use of the material in order to use the correct concentration in the polymer matrix, obtaining a product of reliable quality.

In spite of the polyurethane industry widely using calcium carbonate as a filler, generally the quantity used is defined randomly. Commercial calcium carbonate has large use in this industries because its lower cost, non-toxicity, non-abrasiveness and facilitate pigmentation. In the businesses visited in the region of Belo Horizonte, no data was available on the influence of this filler on the foam, nor was there any methodology to define the ideal quantity of calcium carbonate that should be added without causing damage to the mechanical properties of the final product.

Thus the proposal of the present work was to analyze the morphological and mechanical behavior when various concentrations of commercial calcium carbonate were introduced into the polymer matrix, using scanning electron microscopy (SEM), energy dispersive $\mathrm{X}$ ray spectroscopy (EDS) microanalysis, compressive strength tests in accordance with the ISO 3386-Part 1 standard $^{14}$, thermal analysis (TGA/DTA) and positron annihilation lifetime spectroscopy. In particular, the positron annihilation technique is revealing itself to 
be a promising tool to study the properties of the free volume holes in a non-destructive form.

\section{Experimental}

\subsection{Raw materials}

The raw materials employed were the following: Polyether polyol Voranol 4730N and TDI Voranate T-80 (Dow Chemical); the silicone surfactant PDMS/POE (General Electric); amine Aricat AA 805 (Arinos) and stannous dioctoate II (Miracema-Nuodex) were used as catalysts in the polymerization and expansion reactions ${ }^{15-16}$; distilled water; and commercial calcium carbonate obtained from the mattress manufacturing industries in the region of Belo Horizonte. The isocyanate index used was 132 . A Fisatom model 710 shaft stirring device (power: $25 \mathrm{~W}$, rotation: $25-200 \mathrm{rpm}$ ) was used for stirring. Table 1 shows the formulations employed in this work.

\subsection{Preparation of flexible foams}

Polyol and the filler were placed in a plastic receptacle, and the mixture was stirred until complete homogenization. Next, the amine, surfactant and water were added. The mixture was submitted to mechanical stirring for one minute. Shortly after the catalyst was added and the mixture was stirred again for 30 seconds. After introducing the isocyanate, the mixture was submitted to six seconds of stirring and then poured into a cubical cardboard box $(7 \times 7 \times 7 \mathrm{~cm})$. The foams were left to cure for tree days.

\subsection{Flexible foams analyses}

For the instrumental analyses, the following instruments were used: SEM - Jeol JSM-840;

Microanalysis (EDS) - Jeol-8900 electron probe microanalyzer; mechanical analysis - Lloyd LR5K; thermal analysis - Netzsch STA409EP; positron annihilation sprectroscopy - A fast-fast coincidence spectrometer from Ortec.

SEM: The samples were cut into little pieces $(0.5 \times 0.5 \times 0.5 \mathrm{~cm})$ using scissors. Next they were covered with a fine layer of gold. The samples were analyzed under magnifications of 50, 150 and 500x.

EDS: The samples of calcium carbonate were covered with a fine layer of carbon and analyzed under an accelerating voltage of $15 \mathrm{kV}$ and a current of $20 \mathrm{nA}$.

Mechanical analysis: The specimens taken, $5.0 \mathrm{~cm}$ from the edge, were submitted to four compression-decompression cycles at $70 \%$ of their original height and a velocity of $100 \mathrm{~mm} / \mathrm{min}$ in accordance with the ISO 3386-Part 1 standard. During the fourth cycle the hysteresis was obtained ${ }^{17}$.

Thermogravimetric analysis (TGA): The TGA/DTG curves were performed in the temperature range of $25-950{ }^{\circ} \mathrm{C}$, with samples of approximately $12 \mathrm{mg}$ packed in alumina crucible, and dynamic air atmosphere (100 mL/min gas flow).

Positron annihilation lifetime spectroscopy (PALS): For this measurement, an electronic device called a fast-fast coincidence circuit it was used and consisting basically, of two gamma detectors, two constant fraction differential discriminators (CFDD) and a time-to-amplitude converter (TAC) was used. The measurement of the foams samples were performed with ${ }^{22} \mathrm{Na}$ positron source in sandwich configuration. The calibration of MCA was $50 \mathrm{ps} / \mathrm{chan}-$ nel and the time resolution of the positron lifetime spectrometer measured for $\gamma$ ray of ${ }^{60} \mathrm{Co}$ was $260 \mathrm{ps}$. The analyses by PALS were performed on foams ground in liquid nitrogen. The lifetime spectra were satisfactorily resolved in three components utilizing the POSITRONFIT-EXTENDED program and supplying the values for intensities $\left(\mathrm{I}_{\mathrm{i}}\right)$ and mean lifetime $\left(\mathrm{t}_{\mathrm{i}}\right)$. The subscripts $\mathrm{i}=1,2,3$ refer to the para-positronium (p-Ps), free positron and ortho-positronium (o-Ps) species, respectively ${ }^{18-19}$.

\section{Results and Discussion}

\subsection{Analysis of commercial calcium carbonate}

When a filler is introduced into a polymeric material, the ideal is that it has regular granulometry and that its particles are sufficiently small to enable good distribution in the matrix ${ }^{7}$. Figure 1 presents

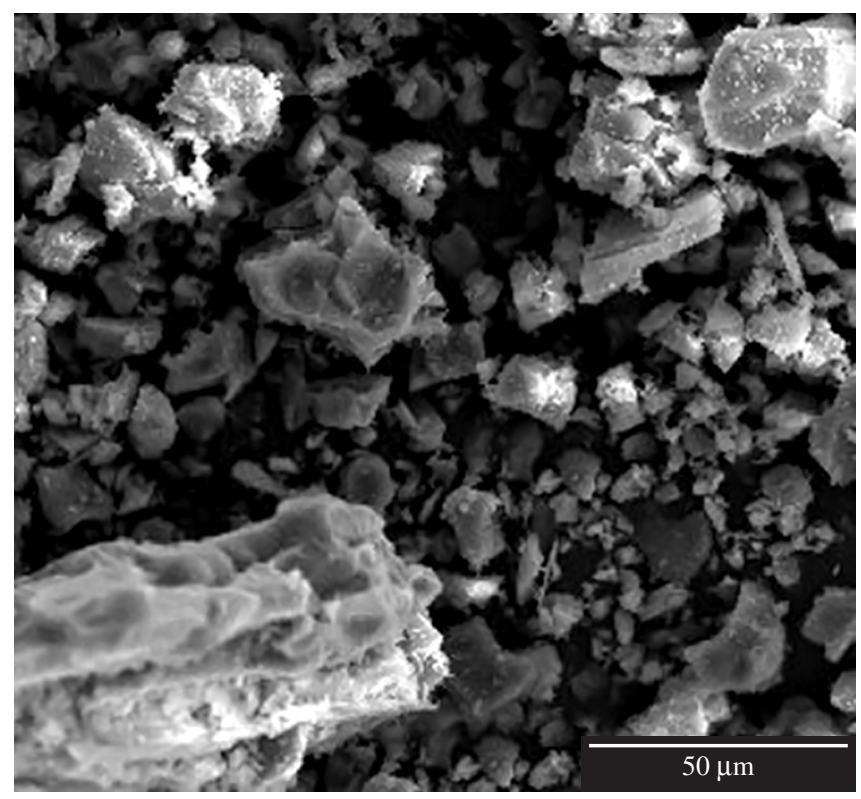

Figure 1. SEM of commercial calcium carbonate (magnification of 500x).

Table 1. Formulations of foams with $\mathrm{CaCO}_{3}$.

\begin{tabular}{|c|c|c|c|c|c|}
\hline $\begin{array}{l}\text { Polymeric } \\
\text { Reagents }\end{array}$ & $\begin{array}{c}\text { Foam with } 1 \% \\
\text { of } \mathrm{CaCO}_{3}(\mathrm{~g})\end{array}$ & $\begin{array}{c}\text { Foam with } 9 \% \\
\text { of } \mathrm{CaCO}_{3}(\mathrm{~g})\end{array}$ & $\begin{array}{l}\text { Foam with } 15 \% \\
\text { of } \mathrm{CaCO}_{3}(\mathrm{~g})\end{array}$ & $\begin{array}{l}\text { Foam with } 21 \% \\
\text { of } \mathrm{CaCO}_{3}(\mathrm{~g})\end{array}$ & $\begin{array}{c}\text { Foam with } 30 \% \\
\text { of } \mathrm{CaCO}_{3}(\mathrm{~g})\end{array}$ \\
\hline Polyol & 30.24 & 30.24 & 30.24 & 30.24 & 30.24 \\
\hline TDI & 15.28 & 15.28 & 15.28 & 15.28 & 15.28 \\
\hline Amine & 0.04 & 0.04 & 0.04 & 0.04 & 0.04 \\
\hline Surfactant & 0.17 & 0.17 & 0.17 & 0.17 & 0.17 \\
\hline Water & 0.90 & 0.90 & 0.90 & 0.90 & 0.90 \\
\hline Catalysts & 0.07 & 0.07 & 0.07 & 0.07 & 0.07 \\
\hline $\mathrm{CaCO}_{3}$ & 0.47 & 4.20 & 7.01 & 9.81 & 14.01 \\
\hline
\end{tabular}

'Total weight of polymeric reagents $=46.70 \mathrm{~g}$; pph $=$ parts per hundred parts of polyol. 
the scanning electron microscopy of commercial calcium carbonate at a magnification of 500x.

It was observed that the calcium carbonate presented irregular, heterogeneous and coarse granulometry. The calcium carbonate, used in the present work, showed a large polydispersion in granulometric analysis with sieves of defined particle sizes. The particle size varied between -100 and +250 mesh. This filler is used in the mattress industry without any pre-treatment because it is a residue generated in the fabrication of lime and sold to the manufacturers at reduced prices.

The calcium carbonate was also submitted to a qualitative $\mathrm{X}$ ray energy dispersive spectrum (EDS, Figure 2), where it was possible to detect the various elements of its constitution. It is worth noting that to improve the foam quality, granulometry aside, the ideal is to utilize a chemically pure material that does not contain substances that could react with the matrix ${ }^{8}$.

\subsection{Analysis of flexible foams}

Figure 3 presents the SEM analysis of the flexible polyurethane foams containing $21 \%$ of calcium carbonate.

It is observed that the calcium carbonate added is agglomerated in some points in the polymer matrix and not evenly distributed ho-

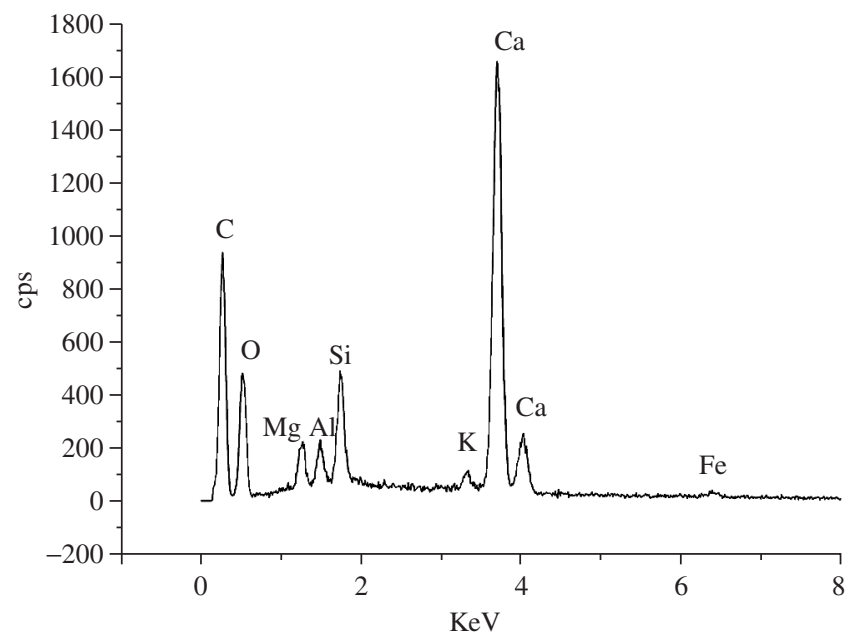

Figure 2. Microanalysis (EDS) of commercial calcium carbonate. mogenously, as is required to promote an efficient reinforcement $t^{5,20}$. Even though the use of excessive quantities of filler causes enrichment, it also is responsible for damage to the matrix (Figure 4) ${ }^{21}$, causing the foam to lose its characteristic polyhedral morphology. The arrows show the worsening morphology as higher concentrations are added.

A low interaction of the filler used and the polymeric matrix was further verified by microscopy (Figure 5), which probably contributed to the deformations seen earlier (Figure 4).

Figure 6 illustrates the thermogravimetric/derivative thermogravimetric (TGA/DTG) curves of the synthesized polyurethane foam and of the commercial calcium carbonate filler. The polyurethane foam presents typical decomposition in two stages between 200 and $600{ }^{\circ} \mathrm{C}$, generating a final residue of $1.73 \%$. The commercial calcium carbonate decomposes in the temperature range $550-760{ }^{\circ} \mathrm{C}$, losing $\mathrm{CO}_{2}$ and generating $\mathrm{CaO}$ with a final residue of $\sim 65.6 \%$. This high value of the residue means that the commercial calcium carbonate, probably, contains inert compounds like $\mathrm{CaO}, \mathrm{SiO}_{2}, \mathrm{Al}_{2} \mathrm{O}_{3}$, etc, as show in the EDS analysis.

\subsection{Mechanical analysis}

In the mechanical analyses, the flexible foams were submitted to four compression-decompression cycles with the objective of evaluating the hysteresis with increasing carbonate concentration ${ }^{14}$. In flexible foams, hysteresis ${ }^{22-23}$ is related to the loss of the energy of the system when it is submitted to a compression-decompression cycle. Hysteresis is a very important property for evaluating the quality of
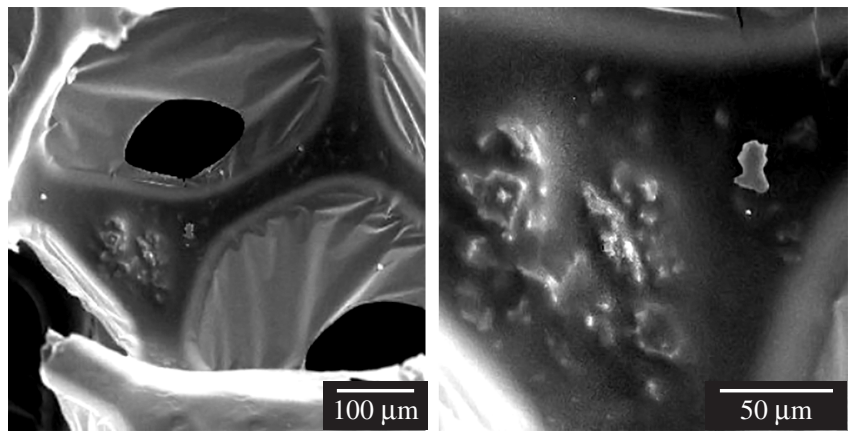

Figure 3. SEM of flexible polyurethane foam filled with $21 \%$ of calcium carbonate (magnification of 150 and 500x).
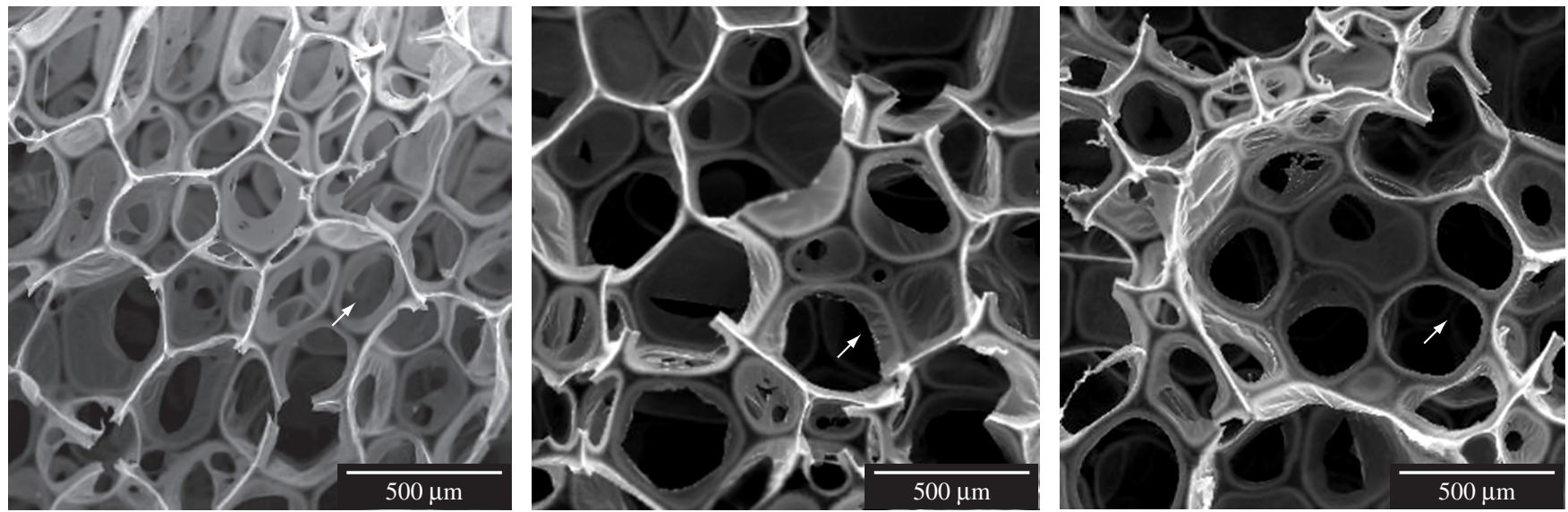

Figure 4. SEM of flexible polyurethane foam filled with carbonate at concentrations of 9, 15 and $21 \%$ (magnification of 50x). 
foams. It is related to the loss of energy caused by the irreversible slippage of the polymer chains in the compression-decompression cycle during use of the foam. The greater the hysteresis, the greater the susceptibility of the material to suffer irreversible deformations ${ }^{13}$.

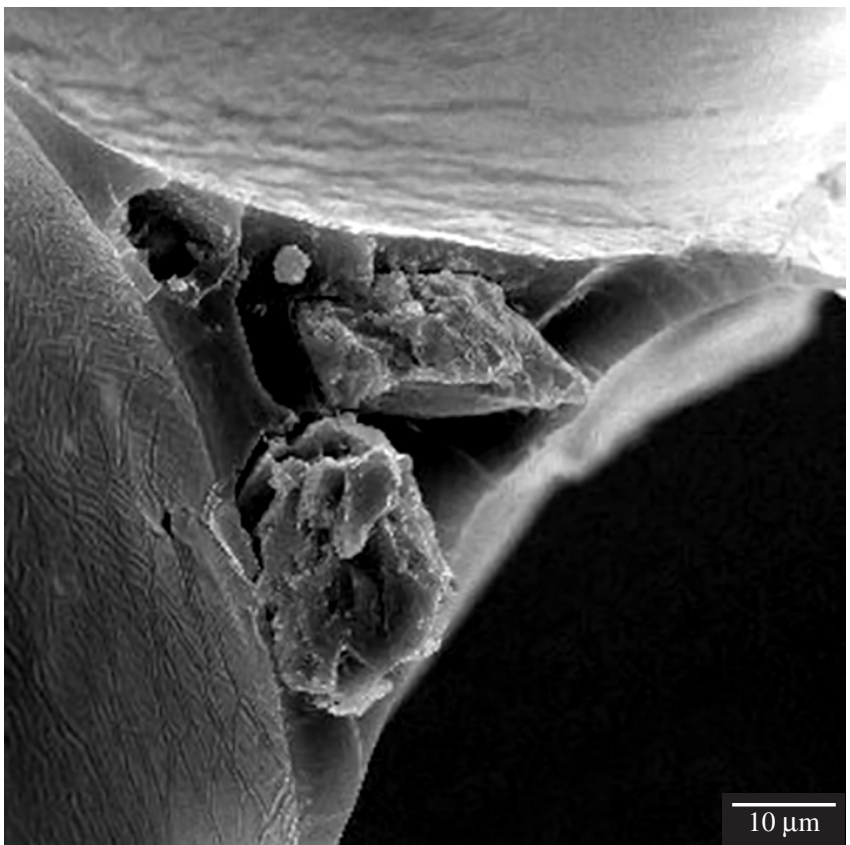

Figure 5. SEM of a transversal section of the flexible polyurethane foam with calcium carbonate (magnification of 1000x).

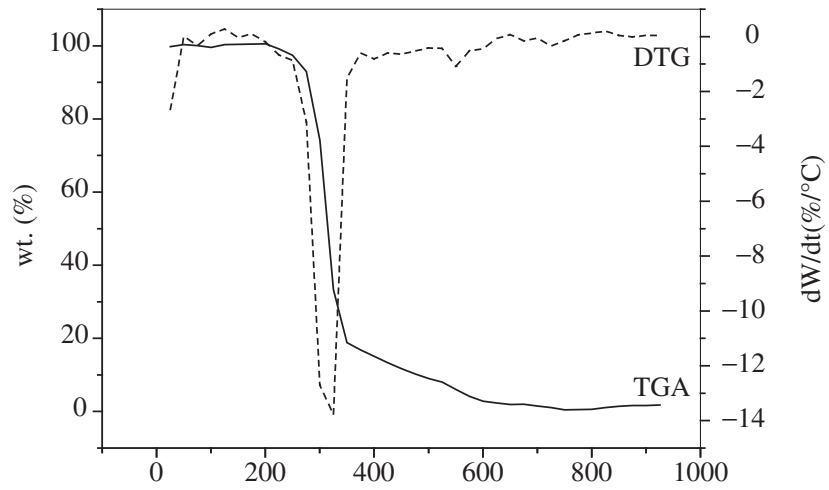

(a)

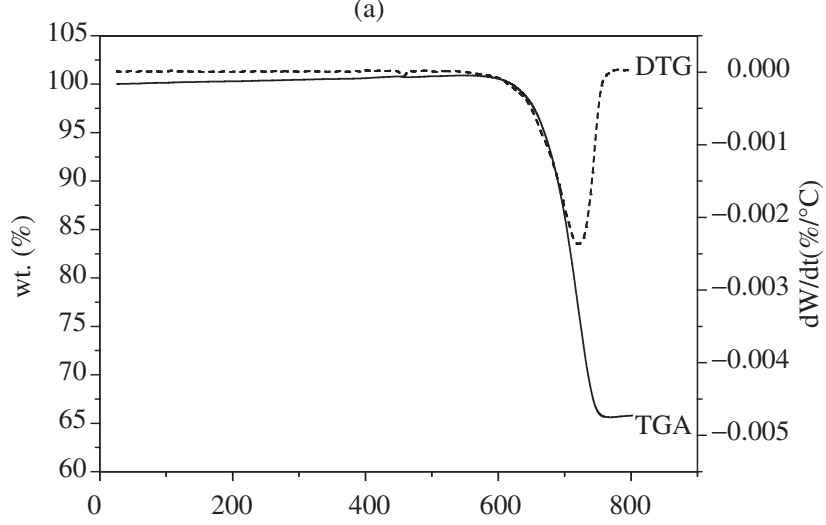

(b)

Temperature $\left({ }^{\circ} \mathrm{C}\right)$

Figure 6. TGA/DTG curves of (a) flexible polyurethane foam and (b) the filler containing commercial calcium carbonate.
As flexible foams are submitted to cycles of compression and decompression in daily use, principally when used in upholstery and mattresses, the measure of hysteresis predicts possible deformations that will influence the quality of the flexible foam ${ }^{2}$. Figure 7 presents the hysteresis values of the foams in compression-decompression tests as a function of increasing filler concentration.

It was observed that increasing the quantity of calcium carbonate in the matrix also increased the hysteresis values, signifying that the system was losing its ability to return to the original shape. Therefore it was concluded that the progressive addition of filler reduced the foam quality since the increased loss of energy during the compression-decompression led to deformation of the matrix. It is worth emphasizing the progressive increase in hysteresis values as a function of the quantity of filler. Hysteresis is also related to the distribution of the filler in the foams. The carbonate is a filler that presents a large surface energy and interaction between the particles, tending to form agglomerates in the matrix ${ }^{24}$. In this manner, the forces are not equally distributed during the compression-decompression, which is also responsible for the deformation exhibited by the foams.

Another important parameter obtained in the mechanical analyses was the compression force deflection at $40 \%$ compression, where the foams were submitted to the compression necessary to cause a deformation of $40 \%$ of the original size. This parameter quantifies the compression that a flexible foam supports without a significant loss in its morphology. The higher the CFD 40 value, the more difficult the compression becomes because the foam is harder and therefore has a greater dimensional stability. Figure 8 shows the graph obtained of CFD 40 as a function of filler concentration for foams with calcium carbonate.

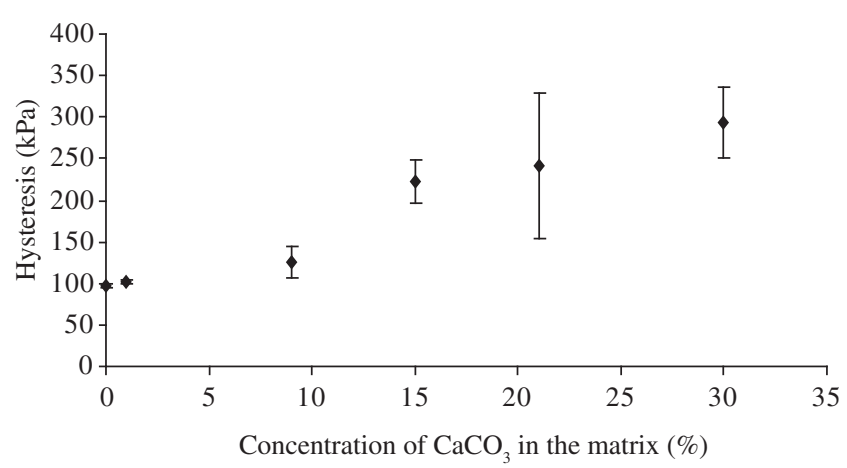

Figure 7. Values of hysteresis as a function of carbonate concentration in the polyurethane matrix.

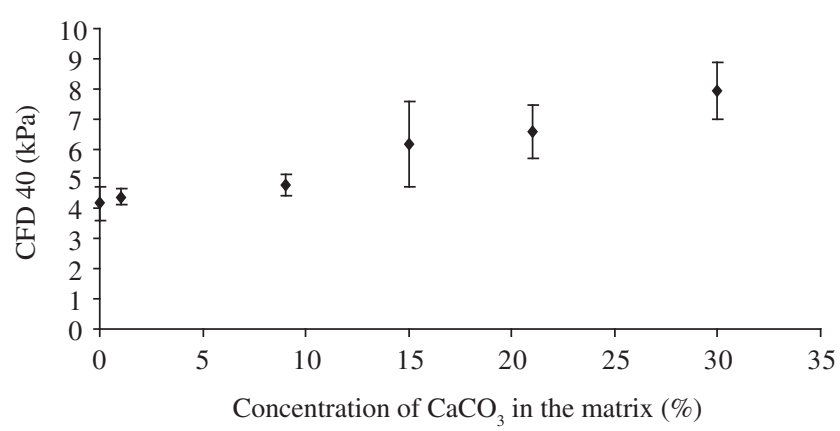

Figure 8. Graph of CFD 40 for flexible polyurethane foams containing commercial calcium carbonate. 
It was observed that the increase in filler concentration increased the foam's hardness, improving the dimensional stability. However, the foam must have an equilibrium between the CFD 40 and hysteresis; in spite of the resulting improved dimensional stability of the foam, the increase of filler favored deformations that lead to a decline in the quality of the material.

\subsection{Positron annihilation lifetime spectroscopy (PALS)}

Polymer science uses the free-volume concept to rationalize many properties at the microscopic structural level, also relating them to macroscopic properties. One valuable method for studying holes with sub-nanometer sizes is positron annihilation lifetime spectroscopy (PALS); it can also be used to determine the size distribution of the holes as well as the free-volume fractions. Inside polymer matrices, thermalized positrons can form positroniums (Ps) that will then be confined to free-volume holes. The main annihilation mechanism of the ortho-positronium (o-Ps; the long-lived triplet state of positronium) is the pick-off annihilation by electrons of the host medium. The annihilation parameters depend on the physicochemical properties. The lifetime and intensity of the o-Ps can then yield the hole size and density ${ }^{25-26}$

In the present work, PALS was utilized to confirm that the calcium carbonate adheres in a mechanical way to the polymer matrix, not having significant interactions between the two materials. For this, two parameters were analyzed: $I_{3}$ and $\tau_{3}$. The parameter $I_{3}$ gives the probability of ortho-positronium formation and is related to the concentration of holes present in the material. The parameter $\tau_{3}$ evaluates the dimensions of the empty spaces in the biphasic material; its variation suggests significant interactions between the constituent materials.

Based on the graph of the variation of $\mathrm{I}_{3}(\%)$ as a function of calcium carbonate concentration in the polymer matrix (Figure 9a), a linear trend was seen that is typical of materials that have their components linked by simple mechanical adhesion ${ }^{19}$. This verification showed that the system presents two phases, signified by the presence of empty spaces created in the interfaces of the polyurethane/ $\mathrm{CaCO}_{3}$ composite.

It was still observed that for the pure components, there is a significant difference that refers to the positronium formation. The pure polyurethane showed significant $(\sim 28 \%)$ positronium formation, while the $\mathrm{CaCO}_{3}(1.9 \%)$ was within the experimental error and did not form positronium. Such a parameter, therefore, is sensitive to the possible interactions between the two components.

Figure $9 \mathrm{~b}$ displays the graph of $\tau_{3}$ as a function of the concentration of calcium carbonate in the polymer matrix. At all concentrations it was observed that the carbonate did not cause a significant variation in $\tau_{3}$, which suggests that the carbonate is only adhering to the matrix by simple mechanical adhesion. Such a parameter, $\mathrm{I}_{3}$, therefore is sensitive to the possible interactions between the two components.

\section{Conclusions}

The calcium carbonate and the polymer matrix present a low interaction that is proportional to mechanical adhesion, as verified by PALS analysis.

The carbonate did not disperse well in the matrix since it has variable particle size and presents interactions that tend to form agglomerates, making it so that there is not a distribution of force between the carbonate and the matrix during the utilization of the flexible foam.

Although the flexible polyurethane foam factories use calcium carbonate as a filler, the introduction of high quantities corroborates with the increase in hysteresis values, leading to a loss in quality.

\section{Acknowledgements}

The authors are grateful to CNPq for financial support and Prof. Dr. José Caetano Machado for the positron annihilation analyses.

\section{References}

1. Ligoure C, Cloitre M, Le Chatelier C, Monti F, Leibler L. Making polyurethane foams from microemulsions. Polymer 2005; 46(17): 6402-6410.

2. Woods G. The ICI Polyurethanes Book. 2 ed. New York: Wiley; 1990.

3. Saliba CC, Oréfice RL, Carneiro JRG, Duarte AK, Schneider WT, Fernandes MRF. Effect of the incorporation of a novel natural inorganic

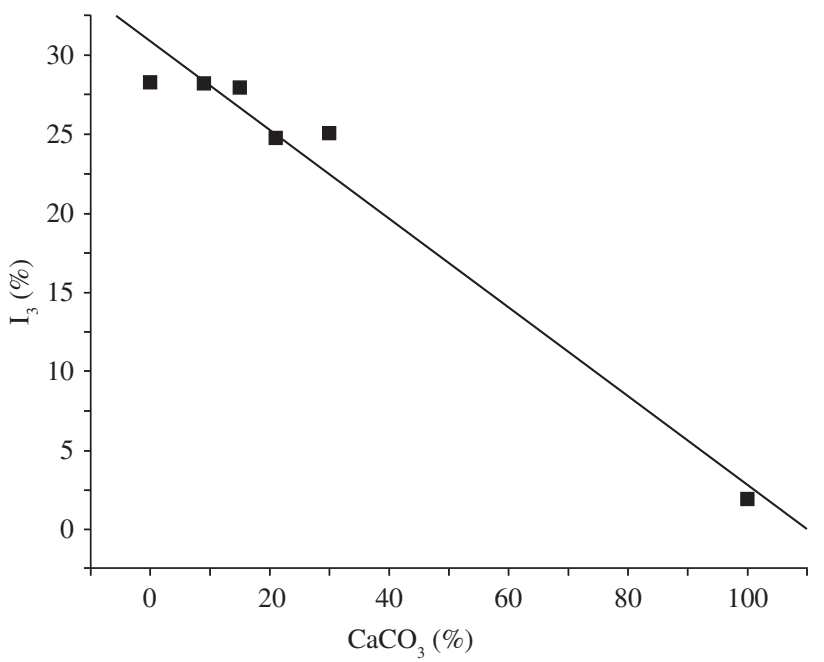

(a)

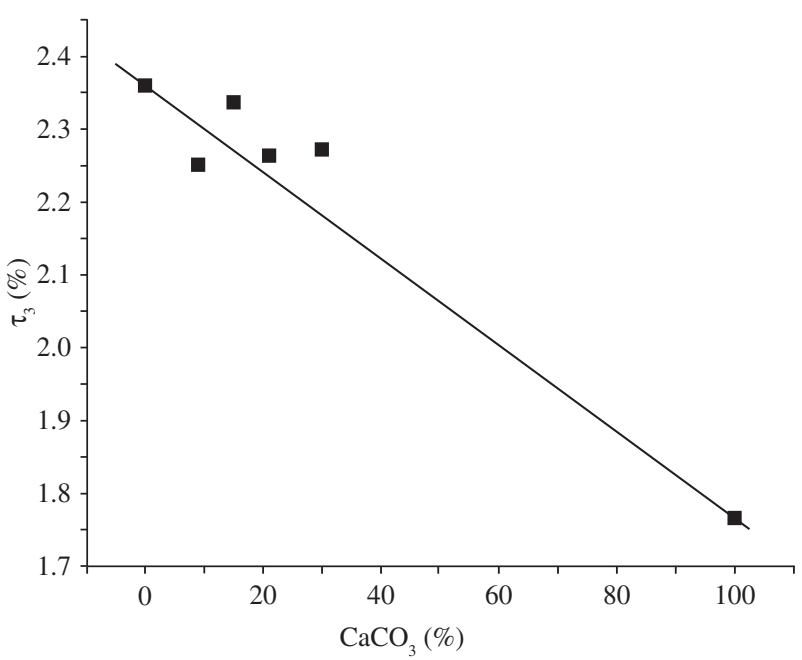

(b)

Figure 9. a) Graph of $\mathrm{I}_{3}(\%)$ as a function of $\mathrm{CaCO}_{3}$ concentration in the flexible polyurethane foams; and b) $\tau_{3}(\%)$ as a function of $\mathrm{CaCO}$ concentration in the flexible polyurethane foams. 
short fiber on the properties of polyurethane composites. Polym. Test. 2005; 24(7): 819-824.

4. Bartczak Z, Argon AS, Cohen RE, Weinberg M. Toughness mechanism in semi-crystalline polymer blends: II. High-density polyethylene toughened with calcium carbonate filler particles. Polymer 1999; 40(9): 2347-2365.

5. Callister WD. Materials Science and Engineering: An Introduction. 5 ed. New York: John Wiley \& Sons; 2000.

6. Nunes RCR, Fonseca JLC, Pereira MR. Polymer-filler interactions and mechanical properties of a polyurethane elastomer. Polym. Test. 2000; 19(1): 93-103.

7. Barma P, Rhodes MB, Salovey R. Mechanical properties of particulatefilled polyurethane foams. J. Appl. Phys. 1978; 49(10): 4985-4991.

8. Rabello M. Aditivação de Polímeros. São Paulo: Artliber; 2000.

9. Saint-Michel F, Chazeau L, Cavaillé JY, Chabert E. Mechanical properties of high density polyurethane foams: effect of the filler size. Composites Science and Technology 2006; 66(15): 2700-2708.

10. Rattanasom N, Saowapark T, Deeprasertkul C. Reinforcement of natural rubber with silica-carbon black hybrid filler. Polym. Test. 2007; 26(3): 369-377.

11. Mothé CG, Araújo CR, Oliveira MA, Yoshida MI. Thermal decomposition kinetics of polyurethane-composites with bagasse of sugar cane. J. Therm. Anal. Calorim. 2002; 67(2): 305-312.

12. Mothé CG, Araújo CR. Properties of polyurethane elastomers and composites by thermal analysis. Thermochim. Acta. 2000; 357-358(14): 321-325.

13. Vilar W. Química e Tecnologia de Poliuretanos. 2 ed. Rio de Janeiro: Vilar Consultoria; 1998.

14. International Organization for Standardization. ISO document 3386-1. Polymeric materials, cellular flexible: Determination of stress-strain characteristics in compression. Part 1, Low-density materials. Geneva; 1986.
15. Neff R, Adedeji A, Macosko CW, Ryan AJ. Urea hard segment morphology in flexible polyurethane foam. J. Polym. Sci., Part B: Polym. Phys. 1998; 36(4): 573-581.

16. Armistead JP, Wilkes GL, Turner RB. Morphology of water-blown flexible polyurethane foams. J. Appl. Polym. Sci. 1998; 35(3): 601-629.

17. Bezazi A, Scarpa F. Mechanical behaviour of conventional and negative Poisson's ratio thermoplastic polyurethane foams under compressive cycling loading. International Journal of Fatigue. 2007; 29(5): 922-930.

18. Porto AO. Estudo de Soluções Sólidas de Complexos Metálicos de Betadicetonatos através da técnica de Aniquilação de Pósitrons. [Thesis]. Belo Horizonte: Universidade Federal de Minas Gerais; 1994.

19. Carvalho CF. Estudo de Misturas e Soluções Sólidas Binárias envolvendo $\beta$-Dicetonatos da Coluna III-A com $\beta$-Dicetonatos de Co(III) e Fe(III) através da técnica de Espectroscopia de Vida Media do Pósitron. [Thesis]. Belo Horizonte: Universidade Federal de Minas Gerais; 1992.

20. Shui M. Polymer surface modification and characterization of particulate calcium carbonate fillers. Appl. Surf. Sci. 2003; 220(1-4): 359-366.

21. Singh RP, Zhang M, Chan D. Toughening of a brittle thermosetting polymer: Effects of reinforcement particle size and volume fraction. $J$. Mater. Sci. 2002; 37(4): 781-788.

22. Yi J, Boyce MC, Lee GF, Balizer E. Large deformation rate-dependent stress-strain behavior of polyurea and polyurethanes. Polymer 2006; 47(1): 319-329.

23. Qi HJ, Boyce MC. Stress-strain behavior of thermoplastic polyurethanes. Mechanics of Materials 2005; 37(8): 817-839.

24. Osman MA, Atallah A, Suter UW. Influence of excessive filler coating on the tensile properties of LDPE - calcium carbonate composites. Polymer 2004; 45(4): 1177-1183.

25. Jean YC, Zhang J, Chen H, Li Y, Liu G. Positron annihilation spectroscopy for surface and interface studies in nanoscale polymeric films. Spectrochim. Acta, Part A. 2005; 61(7): 1683-1691.

26. Marques MFF, Lopes GC, Gordo PM, Kajcsos ZS, Lima AP, Queiroz DP, Pinho MN. Positron studies of temperature-dependence of free volumes in Polydimethylsiloxane/poly(propylene oxide) urethane/urea membranes. Radiat. Phys. Chem. 2003; 68(3-4): 573-576. 